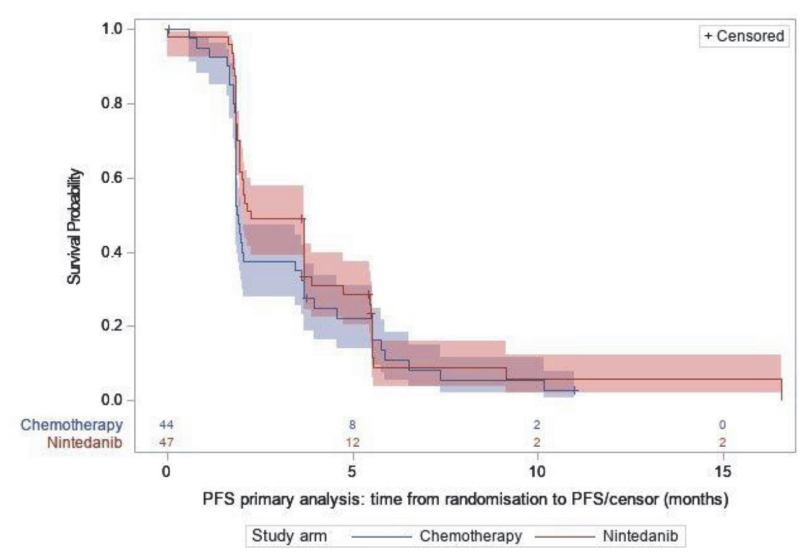

Abstract 596 Figure 1 Progression free survival Kaplan-Meier curves with $80 \%$ Cis

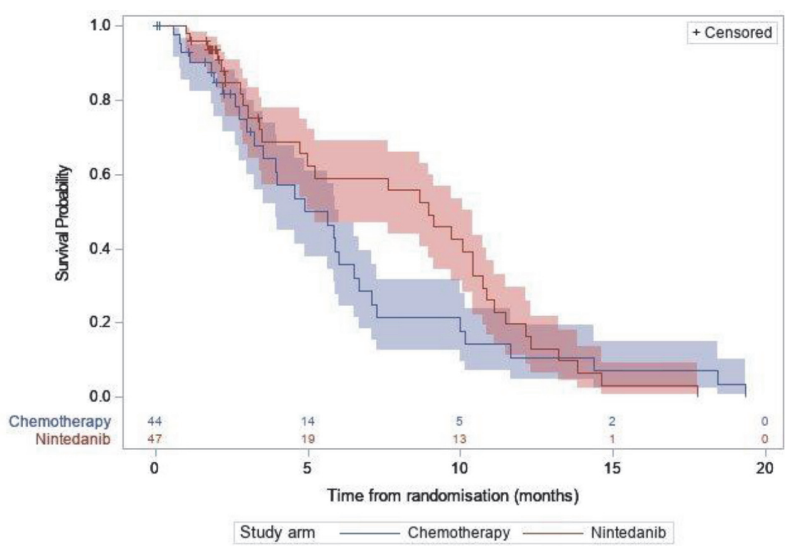

Abstract 596 Figure 2 Overall survival Kaplan-Meier curves, with $80 \% \mathrm{Cls}$

$=0.1521$. Median OS was 9.0 and 4.9 months, respectively. Difference in OS estimates at 6 and 12 months were $19.7 \%$ and $8.9 \%$ demonstrating non-proportional hazards. RR was $2.1 \%$ and $0 \%$, and DCR at 16 weeks was $23.4 \%$ and $9.1 \%$ $($ odds $\quad$ ratio $=5.81, \quad 80 \% \mathrm{CI}=(1.79,18.89), \quad \mathrm{p}(1$-sided $)=0.0276)$ with nintedanib and chemotherapy, respectively.

Conclusion The study failed to demonstrate sufficient activity of nintedanib as a monotherapy to support a phase III trial. However, the benefit in PFS, DCR and OS suggests it may be interesting to combine nintedanib with other agents in OCCC. Chemotherapy is ineffective and the outcomes for women with OCCC are extremely poor confirming the need for continued research into novel targets and therapies. Translational research is on-going (figures 1 and 2).

Disclosures The study was funded by an educational grant from Boehringer Ingelheim and supported by Cancer Research UK Grant ref: C8361/A15600.

Author disclosures Rosalind Glasspool: Grant funding for clinical trials from Boehringer Ingelheim, Lilly/Ignyta and Clovis. Consultancy fees, travel support and/or speaker fees for AstraZeneca, GSK/Tesaro, Clovis, Immunogen and Sotio. Site PI for studies sponsored by AstraZeneca, GSK/Tesaro, Clovis, Immunogen, Lilly and Pfizer.

Samantha Hinsley: none.

Jonathan Ledermann: Advisory Board and Lecture fees from AstraZeneca, Pfizer, Clovis Oncology, MSD/Merck, Eisai,
Artios Pharma, GSK and grant funding from AstraZeneca and MSD/Merck. IDMC fees from Regeneron.

Iain McNeish: Advisory Board fees from AstraZeneca, Clovis Oncology, Tesaro/GSK, Roche, Scancell and Carrick Therapeutics. Institutional grant funding from AstraZeneca.

Jerome Alexandre: Grant funding from Janssen, MSD. Consultancy fees, travel support and/or speaker fees for AstraZeneca, GSK/Tesaro, MSD, Eisai, Novartis, Pharmamar. Site PI for studies sponsored by AstraZeneca, GSK/Tesaro, MSD, Agenus, Merck Serono, Seattle Genetics

Anneke Westermann: none.

Claire Lawless: none

Nelleke Ottevanger: none

Mansoor Raza Mirza: Personal Financial Interests: AstraZeneca, Biocad, Clovis Oncology, Geneos, Genmab, Karyopharm Therapeutics, merck, msd, Oncology Centre, Pfizer, Roche, SeatleGenetics, Sera Prognostics, Sotio, Tesaro-GSK, ZaiLab. Institutional financial interests (study grants): AstraZeneca, Boehringer Ingelheim, Clovis Oncology, Pfizer, Tesaro-GSK

Isabelle Ray-Coquard: Honoraria (self) from Abbvie, Agenus, Advaxis, BMS, PharmaMar, Genmab, Pfizer, AstraZeneca, Roche, GSK, MSD, Deciphera, Mersena, Merck Sereno, Novartis, Amgen, Tesaro and Clovis; honoraria (institution) from GSK, MSD, Roche and BMS; advisory/consulting fees from Abbvie, Agenus, Advaxis, BMS, PharmaMar, Genmab, Pfizer, AstraZeneca, Roche/Genentech, GSK, MSD, Deciphera, Mersena, Merck Sereno, Novartis, Amgen, Tesaro and Clovis; research grant/funding (self) from MSD, Roche and BMS; research grant/funding (institution) from MSD, Roche, BMS, Novartis, AstraZeneca and Merck Sereno; and travel support from Roche, AstraZeneca and GSK.

\section{PERITONEAL CARCINOMATOSIS INDEX AS A PREDICTOR OF CYTOREDUCTIVE OUTCOMES IN PATIENTS WITH ADVANCED OVARIAN CANCER}

${ }^{1}$ Maria Yakovleva, ${ }^{1}$ Olga Smirnova, ${ }^{2}$ Elena Ulrikh, ${ }^{1}$ Elvina Bakaeva, ${ }^{1}$ Anna Petrova, ${ }^{1}$ Ksenia Kozyreva, ${ }^{1}$ Lev Shevkunov, ${ }^{1}$ Nikolay Mikaya, ${ }^{1}$ Adel Urmancheeva, ${ }^{1}$ Igor Berlev. ${ }^{1} N$. N. Petrov National Medical Research Center of Oncology; ${ }^{2}$ Almazov National Medical Research Centre; North-Western State Medical University, N.N.Petrov National Medical Research Centre of Oncology; Oncology

\subsection{6/ijgc-2020-ESG0.224}

Introduction/Background In our study, we evaluated the peritoneal carcinomatosis index (PCI) and to study the influence of PCI on the cytoreductive surgery outcomes in patients with advanced ovarian cancer.

Methodology We analyzed the results of 133 patients with serous high-grade ovarian cancer FIGO IIIC-IVB stages. PCI was evaluated at 13 regions (score $0-3$ for each region) via pelvic MRI, chest and abdominal CT and staging laparoscopy (S-LPS.)

Results Were performed 23\% (31/133) of primary and $77 \%$ $(102 / 133)$ of interval debulking surgery (DS), 68\% (90/133) of them were optimal (no residual disease, ODS) and 32\% (43/ 133) were suboptimal (residual tumor of $>1 \mathrm{~cm}$, SDS). The frequency of ODS for PCI $0-15$ score was $60-86 \%$, for PCI 15-20 score - 9\%, PCI> 20 score - 0\%. Preoperative and intraoperative determination of the PCI was equal in 58\%. In $19.8 \%$ PCI was overestimated during preoperative examination, while the use of laparoscopy evaluated PCI allowed to perform an ODS in these cases. In 21\% PCI was 
underestimated and S-LPS helped to avoid SDS in $89 \%$ because of noting a diffuse bowel mesentery involvement. Conclusion A combination of MRI/CT scans and staging laparoscopy in PCI evaluating is an effective treatment modality which can improve the cytoreductive outcomes in patients with advanced ovarian cancer.

Disclosures No conflict of interest exits in the submission of this study. We confirm that no funding source were used in this study.

\section{Prevention of gynaecologic cancer}

\section{EARLY SALPINGECTOMY (TUBECTOMY) WITH DELAYED OOPHORECTOMY AS AN ALTERNATIVE FOR RISK- REDUCING SALPINGO-OOPHORECTOMY TO IMPROVE QUALITY OF LIFE IN WOMEN WITH A BRCA $1 / 2$ PATHOGENIC VARIANT (TUBA STUDY): A PROSPECTIVE MULTICENTER PREFERENCE TRIAL}

${ }^{1}$ Miranda P Steenbeek, ${ }^{1}$ Marline Harmsen, ${ }^{2}$ Nicoline Hoogerbrugge, ${ }^{3}$ Tuba Study Group, ${ }^{4}$ Rosella PMG Hermens, 'Joanne A de Hullu. 'Radboudumc; Obstetrics and Gynecology; ${ }^{2}$ Radboudumc; Clinical Genetics; ${ }^{3}$ Radboudumc; ${ }^{4}$ Radboudumc; Scientific Institute for Quality of Healthcare

10.1136/ijgc-2020-ESG0.225

Introduction/Background Currently, risk-reducing salpingooophorectomy (RRSO) around the age of 40 is recommended to women with a BRCA1/2 pathogenic variant (PV). To prevent premature menopause, risk-reducing salpingectomy (RRS) is considered, because recent data indicate the Fallopian tube instead of the ovary as origin of high grade serous ovarian carcinoma (HGSC). Based on this hypothesis, the TUBA study (NCT02321228) compares quality of life (QoL) between the novel RRS with delayed oophorectomy (RRO) and standard RRSO.

Methodology Within this national multicenter preference trial, BRCA1/2-PV carriers chose between the novel strategy (RRS upon completion of childbearing and RRO at age 40-45 (BRCA1) or 45-50 (BRCA2)) and the standard strategy (RRSO at age 35-40 (BRCA1) or 40-45 (BRCA2)). The primary outcome is menopause-related QoL, measured by the Greene climacteric scale (GCS). A higher sum of the GCS represents more menopausal complaints.

Results A total of 577 women were included, 51.5\% carried a BRCA1-PV, and 72\% chose the novel RRS with delayed RRO. Until now, 394 women underwent RRS and 154 RRSO of which $30 \%$ did not start hormone replacement therapy (HRT). Without HRT, the adjusted mean increase from baseline on the GCS was 0.6 points (95\% confidence interval (CI) $0.0 ; 1.1$ ) one year after RRS and 7.7 points (95\% CI 6.2;9.9) one year after RRSO. Thus, the adjusted mean difference between the treatment groups was 7.2 (95\% CI 5.4;9.0, $\mathrm{P}<0.001)$. In women with HRT after RRSO, a difference of 3.4 points (95\% CI 2.2;4.6, $\mathrm{P}<0.001)$ was found compared to RRS. For sexual functioning, women without HRT had an increase of 0.4 points $(95 \%$ CI $-0.3 ; 1.1)$ one year after RRS and a decrease of 5.7 points $(95 \%$ CI $-8.7 ;-3.7)$ one year after RRSO. A decrease of 1.6 points (95\% CI -3.2;0.0) was found one year after RRSO with HRT. A decrease represents a worsening of sexual functioning. No differences in cancer worry, decisional conflict or decisional regret were found between groups. No HGSC has occurred during follow-up.
Conclusion Menopause-related QoL is better after novel RRS when compared to RRSO in women with a BRCA1/2-PV, regardless of HRT use. Moreover, sexual functioning is better at one year after RRS. No cancers have occurred since RRS, but follow-up is too short to draw conclusions on safety. An international follow-up study is currently recruiting to evaluate the oncological safety of RRS with delayed RRO (TUBA-WISP II, NCT04294927).

Disclosures Nothing to disclose.

\section{THE ROLE OF HPV GENOTYPES ANALYSIS TO PREVENT OVERTREATMENT OF HIGH-GRADE CIN}

M Alessia Salamina, Massimiliano Fambrini, Francesca Malentacchi, Milo Giani, Federica Barsanti, Irene Renda, Flavia Sorbi. Department of Experimental, Clinical and Biomedical Sciences, University of Florence, Viale G.B. Morgagni, 50, Florence, Italy Careggi University Hospital

\subsection{6/ijgc-2020-ESG0.226}

Introduction/Background Invasive Cervical Cancer (ICC) develops from Cervical Intraepithelial Neoplasia (CIN) and persistence of high-risk HPV (HR-HPV) infection represents the main risk factor for evolution of CIN into ICC. Prevention of the disease is based on two screening methods: Papanicolau Smear (Pap-test) and HPV test; the latter with higher sensitivity and lower specificity. HR-HPV positive test detects earlier than Pap-test high-grade CIN (CIN 2-3) at colposcopy-guided biopsy, but the subsequent conization often reveals the absence of an high-grade CIN. The objective of the present study is to assess the incidence of persistency of high-grade CIN at conization after a positive biopsy. Secondly we aimed to investigate the role of HPV genotypes in the evolution of the lesion, in order to better triage women at time of colposcopy.

Methodology A prospective study was conducted at Local Health Unit Toscana Centro and Careggi University Hospital (Florence, Italy) between January 2016 and February 2017 involving 308 women undergoing conization for high-grade CIN at cervical biopsy. All recruited patients underwent HPV testing prior conization. Biopsy and cone specimen data were recorded for each patient.

Results Only in half of the patients there was a persistency of high-grade CIN. Histology discrepancy between biopsy and cone was observed in 181 out of the 308 recruited patients $(58.7 \% ; \mathrm{p}<0,001)$. There was evidence of a $37.4 \%$ of regression of CIN grade at cone, a $41.2 \%$ of stability and a $21.4 \%$ of progression. The overall evaluation of CIN evolution (regression, stability, progression) and HPV genotypes distribution showed a significant difference depending on HPV positive/negative samples $(\mathrm{p}<0.001)$, HPV risk $(\mathrm{p}=0.005)$ and genotype $(\mathrm{p}<0.001)$. HR-HPVs were highly represented in progression, being HPV16 genotype strikingly prevalent in this group, and significantly lower in patients with regression of the lesion.

Conclusion Approximately a third of patients with high-grade CIN at biopsy detected with Paptest and HPV screening underwent overtreatment with conization. Absent or LR HPV infection at time of conization were identified as predictors of regression. HPV test before conization could triage patients with high-grade CIN at cervical biopsy towards conisation or further follow-up with colposcopy.

Disclosures The authors declare that there is no conflict of interest. 